\title{
A Synchronous Metastatic Growth in Thyroid Gland from Carcinoma Cervix
}

\author{
Chanchal Rana ${ }^{1}$, Pooja Ramakant ${ }^{2}$, Kulranjan Singh ${ }^{3}$, Suresh Babu ${ }^{4}$, Anand Mishra ${ }^{5}$, Preeti Khokhar ${ }^{6}$
}

\begin{abstract}
Aim: To describe a rare case of synchronous carcinoma cervix metastasizing to the thyroid, while the patient being of therapy for primary cancer. Background: Metastasis to thyroid primarily occurs from lung, kidney, gastrointestinal tract (GIT), and head and neck. Cervix cancer metastasis to thyroid had been rarely reported.

Case description: We report an unsealed case of carcinoma cervix metastasizing to the thyroid gland, while being on concurrent chemoradiotherapy for primary malignancy and total thyroidectomy was performed as palliative management. Pathological examination confirmed the thyroid lesion as metastasizing squamous cell carcinoma from the cervix. The postoperative period was uneventful and the patient was referred to radiotherapy department but later developed thrombosis in major neck veins and developed the superior vena cava syndrome. The patient finally succumbed to the disease after 3 weeks.

Conclusion: Very few cases of cervix cancer metastasis to thyroid have been reported so far. Synchronous metastasis in such cases is even rarer. Hence, any patients who presents with palpable nodules in the thyroid and have a history of a previous malignancy must be considered for metastatic disease and in female patients the possibility of metastasis from the cervix should be considered and managed as well as evaluated accordingly.

Clinical significance: Synchronous metastatic growth in the thyroid is known to have a poor prognosis and although total thyroidectomy is not recommended in metastatic disease, but can be performed as a part of palliative treatment.

Keywords: Cervical carcinoma, Metastasis, Synchronous, Thyroid.

World Journal of Endocrine Surgery (2019): 10.5005/jp-journals-10002-1266
\end{abstract}

In spite of having rich vascular supply metastases to the thyroid is less common and account for $1.4-3 \%$ of all the thyroid malignancies. 'Commonly these metastases arise from cancer of lungs, kidney, melanoma, gastrointestinal tract, as well as head and neck. 'Metastasis from squamous cell carcinoma has not been widely reported. ${ }^{1,2}$ We describe one such interesting case when the metastasis to thyroid developed synchronously with the primary malignancy while the patient was on chemoradiotherapy.

\section{Case Description}

A 48-year-old woman presented with a swelling in front of the neck for 15 days. The swelling was painful and rapidly increasing in size. The patient also complained of difficulty in swallowing and breathing. She was diagnosed as a patient with nonkeratinizing squamous cell carcinoma cervix for which she received chemotherapy (paclitaxel and cisplatin) and was receiving the last \# of radiotherapy when she noticed thyroid swelling. She did not have any other comorbidity. On examination, she had multinodular goiter which was $3 \times 4$ $\mathrm{cm}$ involving both lobes and was hard and more on the left lobe. Associated levels II-V cervical lymphadenopathy was also observed.

Ultrasonography (USG) neck revealed enlarged and nodular left lobe with irregular outline. Multiple left-side enlarged lymph nodes were also seen near the jugular vein. Computed tomography (CT) scan neck showed a thyroid with multinodular mass with multiple bulky nodular masses infiltrating esophagus, encasing left common carotid artery, and internal jugular vein. Left-sided multiple lymph nodes enlarged lymph nodes were also seen (Fig. 1). An 18 fluorodeoxyglucose (FDG) PET CT scan showed a heterogeneously FDG avid and contrast enhancing soft tissue density mass lesion, left lobe more than right
1,4,6 Department of Pathology, King George's Medical University, Lucknow, Uttar Pradesh, India

2,3,5 Department of Endocrine Surgery, King George's Medical University, Lucknow, Uttar Pradesh, India

Corresponding Author: Chanchal Rana, Department of Pathology, King George's Medical University, Lucknow, Uttar Pradesh, India, e-mail: chanchal11aug@yahoo.com

How to cite this article: Rana C, Ramakant P, Singh $\mathrm{K}$, et al. A Synchronous Metastatic Growth in Thyroid Gland from Carcinoma Cervix. World J Endoc Surg 2019;11(3):82-85.

Source of support: Nil

Conflict of interest: None

thyroid lobe measuring approximately (4.5 AP $\times 5.2 \mathrm{TR} \times 8 \mathrm{CC}$, SUV max-10.2). The tracheal deviation was to the right side and fat planes with trachea were obscured.

Fine-needle aspiration cytology (FNAC) was performed. These smears were highly cellular and showed clusters as well as the singly scattered population of atypical cells in a hemorrhagic background (Fig. 2). These atypical cells showed high $\mathrm{n} / \mathrm{c}$, moderately anisomorphic hyperchromatic nuclei, coarse chromatin, prominent nucleoli, and scant cytoplasm. These smears were reported as positive for malignancy and with this morphology differential diagnosis of anaplastic carcinoma thyroid, poorly differentiated carcinoma thyroid, and metastatic carcinoma were considered. Hence, a core needle biopsy was done and it revealed diffuse infiltration by sheets of atypical cells. These cells had a high nucleocytoplasmic ratio, moderately anisomorphic hyperchromatic nuclei, conspicuous nucleoli, and scant cytoplasm (Fig. 3). With the above-mentioned differential diagnosis in

(0) The Author(s). 2019 Open Access This article is distributed under the terms of the Creative Commons Attribution 4.0 International License (https://creativecommons. org/licenses/by-nc/4.0/), which permits unrestricted use, distribution, and non-commercial reproduction in any medium, provided you give appropriate credit to the original author(s) and the source, provide a link to the Creative Commons license, and indicate if changes were made. The Creative Commons Public Domain Dedication waiver (http://creativecommons.org/publicdomain/zero/1.0/) applies to the data made available in this article, unless otherwise stated. 


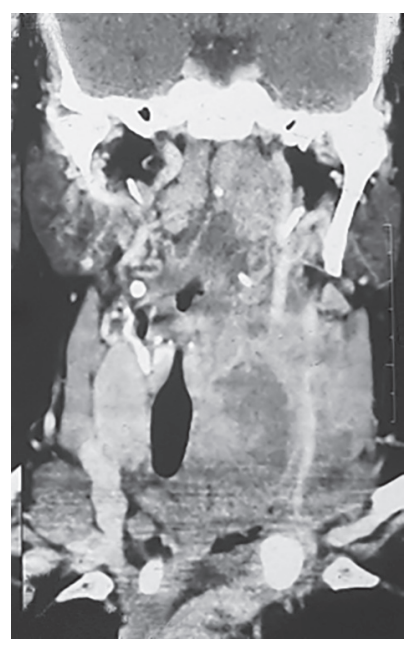

Fig. 1: Computed tomography scan neck showing the thyroid nodules and encased carotid sheath by bulky left side nodal mass

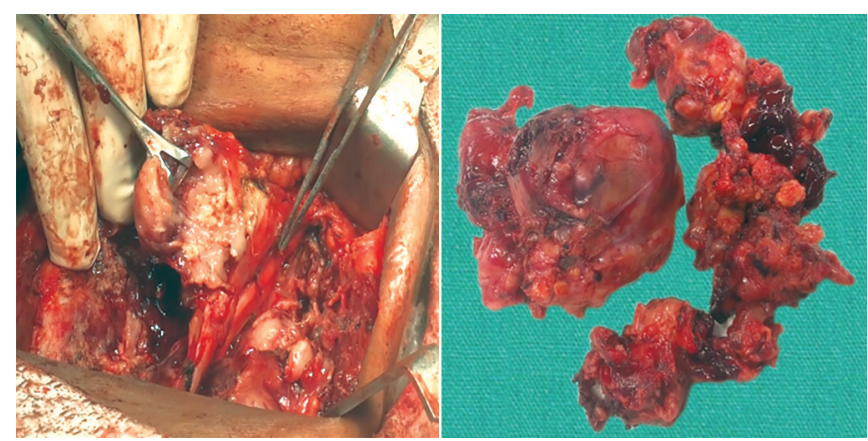

Fig. 3: Intraoperative picture showing the entire carotid sheath and nodal masses medial and lateral to it and total thyroidectomy with central compartment and left modified radical neck dissection specimen

mind, immunohistochemistry was performed. The tumor cells showed diffuse cytoplasmic positivity for vimentin as well as cytokeratin and were negative for thyroglobulin. Some more makers were planned but could not be performed as the tissue was small and got exhausted. Although with the given panel, the primary thyroid malignancy could be ruled out because the tumor cells were negative for thyroglobulin. The possibility of metastatic carcinoma was considered.

Because of the swelling was causing severe pain, discomfort, and dysphagia to the patient, a palliative surgery was performed and total thyroidectomy with central lymph node compartment dissection and left side modified radical neck dissection performed (Fig. 4). Representative sections were taken and processed for histopathological evaluation. The sections showed an infiltrating neoplasm composed of sheets of atypical cells. The main bulk was present at the periphery and the sheets of tumor cells were seen infiltrating the thyroid parenchyma such that thyroid follicles were seen between the tumor cells. These tumor cells had similar morphology as described in the core needle biopsy specimen. Apart from infiltrating tumor cells, there was a background of nodular goiter, which was characterized by the presence of multiple variable-sized nodules composed of colloid-filled follicles lined by flattened to the cuboidal epithelium. These findings were consistent with metastatic carcinoma, but for identification of primary site immunohistochemistry was performed (Fig. 5). Apart from immunohistochemistry (IHC) markers done in core needle biopsy, additional markers were performed. These tumor cells showed

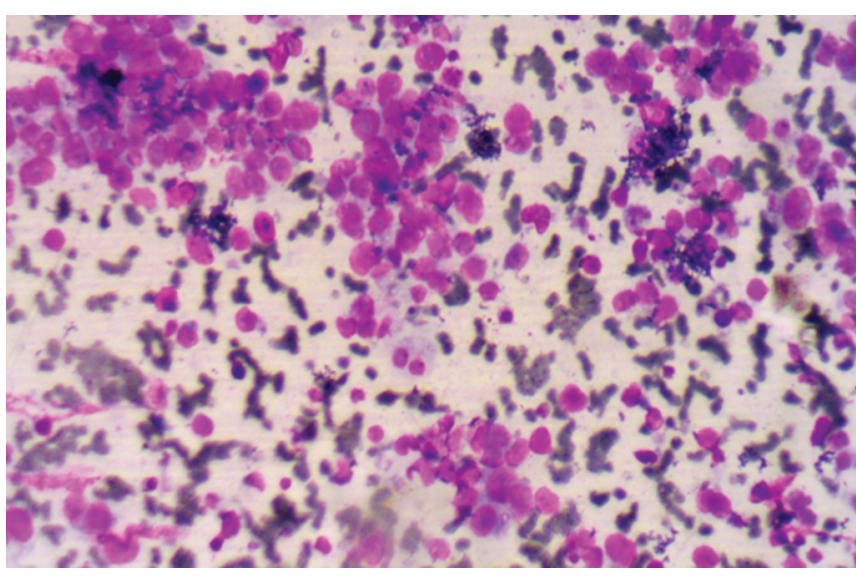

Fig. 2: Cytology smears (Giemsa staining) displaying small clusters as well as scattered population of atypical cells with pleomorphic hyperchromatic nuclei, coarse chromatin, prominent nucleoli, and scant to moderate cytoplasm

diffuse positivity for p53 and were negative for thyroglobulin transcription factor 1 (TTF-1) (Fig. 5).

Hence, after complete analysis, this case was diagnosed as metastatic squamous cell carcinoma thyroid from the cervix. The postoperative period was uneventful and she was transferred to Radiation Therapy Department for chemotherapy and radiation therapy to neck but she developed thrombosis in major neck veins and developed the superior vena cava syndrome. She deteriorated in spite of all palliative measures and finally succumbed to the disease after 3 weeks.

Willis first described metastatic tumors in thyroid in $1931 .^{3}$ The possible mechanism might be the presence of high oxygen and iodine concentration along with high intrathyroidal vascular flow, which may impair the ability of tumor emboli to settle, adhere, and implant. ${ }^{4}$ Metastases to thyroid gland from cervix cancer is very rare, such that $<10$ cases have been described in literature so far. The metastatic tumor can be synchronous or metachronous and the patient with the metachronous disease is known to have better survival. ${ }^{5}$ The mean latency between diagnosing primary malignancy and its metastases is longest for sarcoma ( 70 months) and shortest in lung cancer (4.5 months). ${ }^{1}$ In our patient, the metastatic disease developed when she was receiving her last fraction of radiotherapy, thereby making it a synchronous growth.

Distant metastases in cervical cancer arise primarily by the lymphatic spread. Hematogenous spread is relatively uncommon and is presumed to occur via the blood stream to the caval venous system. They develop either due to the metaplastic transformation of follicular epithelial cells or from a remnant of the thyroglossal duct wall. ${ }^{5}$ In case of primary squamous cell carcinoma (SCC), thyroid neoplastic cells arise from thyroidal cells and this transformation is noticed histologically, while in this study thyroidal component was well demarcated from the neoplastic cells suggesting metastatic infiltration. Immunohistochemically, negative staining with thyroglobulin, TTF-1, and calcitonin ruled out primary malignancy. In patients with an oncological history and a rapidly increasing thyroid nodule, possibility of metastasis should always be considered.

Treatment depends on whether there are solitary metastases to the thyroid or is it part of generalized disseminated disease. In solitary metastases, surgical excision has proven to improve survival rates. In case of multiple site metastases, chemotherapy with/ 




Figs 4A to D: (A) Trucut biopsy displaying diffuse infiltration by tumor cells which are shown (B) diffuse cytoplasmic positivity for vimentin and are (C) negative for TTF-1 and (D) thyroglobulin on immunohistochemistry

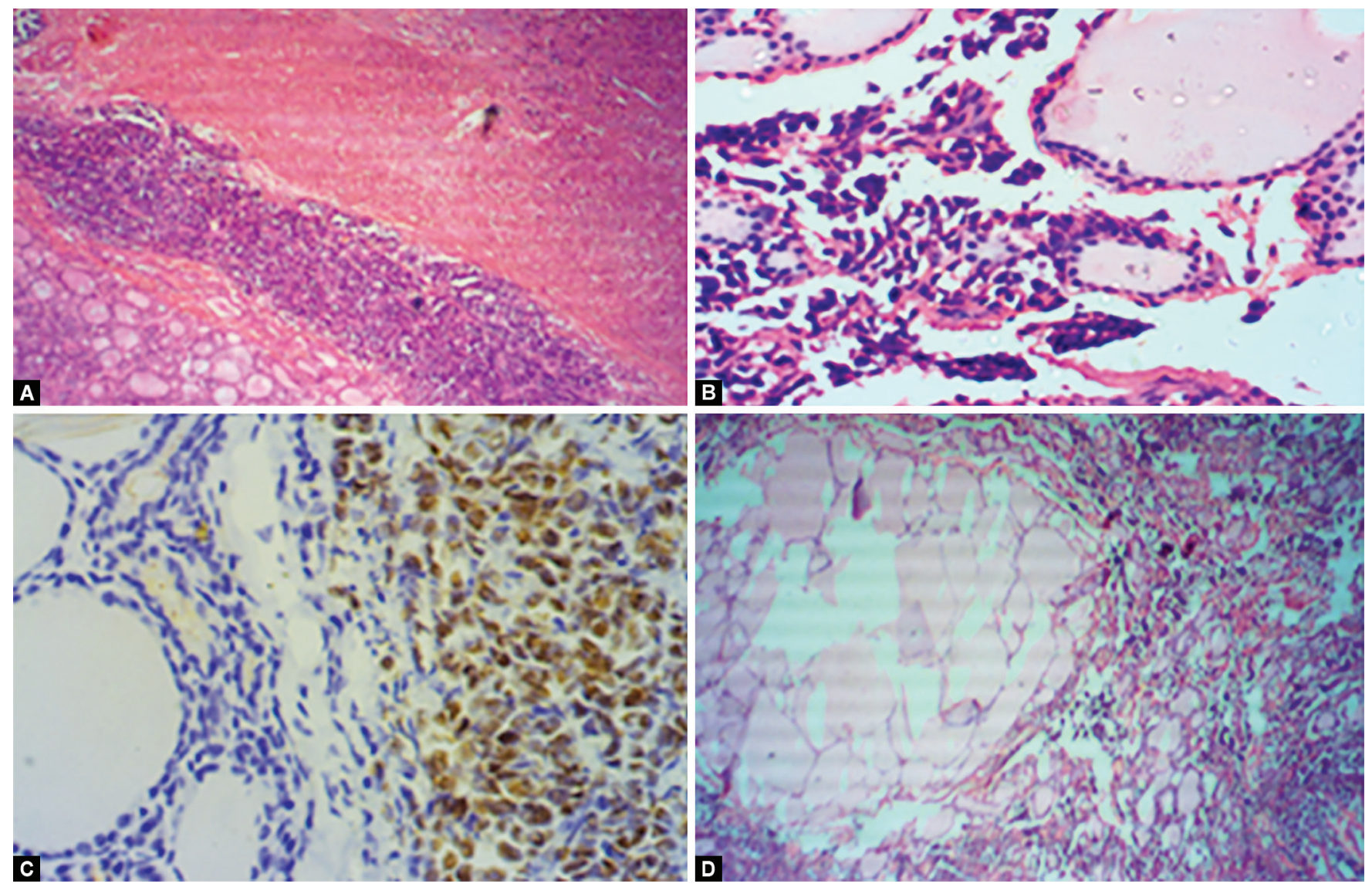

Figs 5A to D: (A) Tumor cells in sheets demarcated from normal appearing thyroid follicles; (B) Tumor cells infiltrating the thyroid parenchyma inbetween the follicles; (C) Tumor cells are positive for p63 on immunohistochemistry; (D) Intervening thyroid showing a background of nodular goiter 
without locoregional radiation therapy is recommended. ${ }^{2}$ Overall, the prognosis of such lesions is very poor in spite of all aggressive management protocols.

\section{References}

1. Celik SU, Besli D, Sak SD, et al. Thyroid gland metastasis from cancer of the uterine cervix: an extremely rare case report. Acta Medica (Hradec Kralove) 2016;59(3):97-99. DOI: 10.14712/18059694. 2016.97.
2. Karapanagiotou E, Saif MW, Rondoyianni D, et al. Metastatic cervical carcinoma to the thyroid gland: a case report and review of the literature. Yale J Biol Med 2006;79(3-4):165-168.

3. Willis RA. Metastatic tumors in the thyroid gland. Am J Pathol 1931;7(3):187-208.

4. Montero PH, Ibrahimpasic T, Nixon IJ, et al. Thyroid metastasectomy. J Surg Oncol 2014;109(1):36-41. DOI: 10.1002/jso.23452.

5. Vamsy M, Dattatreya SP, Sarma LY, et al. Metastatic squamous cell carcinoma thyroid from functionally cured cancer cervix. Indian J Nucl Med 2013;28(2):112-114. DOI: 10.4103/0972-3919.118252. 